\title{
Transport of (Glyco)Sphingolipids in and Between Cellular Membranes; Multidrug Transporters and Lateral Domains
}

\author{
Gerrit van Meer, ${ }^{1,2}$ Dan Sillence, ${ }^{1}$ Hein Sprong, ${ }^{1}$ Nanette Kälin, ${ }^{1}$ and René Raggers ${ }^{1}$
}

Sphingolipids are highly enriched in the outer leaflet of the plasma membrane lipid bilayer. However, the first glycolipid, glucosylceramide, is synthesized in the opposite, cytosolic leaflet of the Golgi membrane. This has led us to experiments which suggest that the level of glucosylceramide in the cytosolic surface is carefully regulated both by the balance between synthesis and hydrolysis and by transport away from this surface through translocators, multidrug transporters, the same molecules that make cancer cells resistant to chemotherapy. Our data suggest a role for newly synthesized glucosylceramide not only in the formation of domains in the luminal leaflet of the Golgi but also on the cytosolic surface of this organelle.

KEY WORDS: Glycolipids; sphingolipids; multidrug resistance; MDR1; MRP1; translocation; transmembrane; flippase; flip.

ABBREVIATIONS: BB, benzbromarone; BFA, brefeldin A; BSA, bovine serum albumin; Cer, ceramide; GlcCer, glucosylceramide; MDR1, the multidrug resistance transporter 1; MRP1, the multidrug resistance protein 1; NBD, C6-NBD-, N-6[7-nitro2,1,3-benzoxadiazol-4-yl]-amino-hexanoyl; PC, phosphatidylcholine; Pgp, P-glycoprotein; SM, sphingomyelin; TGN, trans Golgi network.

\section{INTRODUCTION}

Sphingolipids are thought to be mainly localized on the outside of the plasma membrane, where they exert functions in cell-cell interactions and in modulation of cell surface proteins (Hakomori and Igarashi, 1995). An example of the latter is that sphingolipids are thought to cluster with cholesterol into domains that bring together various components of a machinery that transduces signals from the outside towards the cytoplasm (Lisanti et al., 1994; Okamota et al., 1998; Varma and Mayor, 1998). Similar domains had been proposed to function in the luminal leaflet of the Golgi in the sorting of lipids and membrane proteins from the trans-Golgi network (TGN) to the apical surface of epithelial cells (van Meer et al., 1987; Lisanti and Rodriguez-Boulan, 1990; Keller and Simons, 1998).

While these ideas are in line with the synthesis of the complex glycosphingolipids and sphingomyelin (SM) on the luminal aspect of Golgi membranes, a complication was caused by the finding that synthesis of the simple glycosphingolipid

\footnotetext{
${ }^{1}$ Department of Cell Biology and Histology, Academic Medical Center, University of Amsterdam, PO Box 22700, 1100 DE Amsterdam, The Netherlands. Email: g.vanmeer@amc-uva.nl

${ }^{2}$ To whom correspondence should be addressed.
} 
glucosylceramide (GlcCer) occurs on the opposite, cytosolic side of the Golgi (Coste et al., 1986; van Meer, 1998). GlcCer can translocate across the membrane of the Golgi to be converted to higher glycolipids in the lumen of the Golgi or to be transported on the luminal side of transport vesicles to the cell surface, i.e., the outer leaflet of the plasma membrane (Lannert et al., 1994; Burger et al., 1996). However it is unclear what fraction of the newly synthesized GlcCer translocates across the Golgi membrane vs. what fraction is transported to the cytosolic surface of other organelles. In addition, it is unclear whether newly synthesized GlcCer can also reach the cell surface by direct translocation across the plasma membrane. GlcCer occurs in every cell and is especially abundant in epithelial cells. The question was asked whether GlcCer transport to the cell surface would be blocked in the absence of vesicular transport.

\section{MATERIALS AND METHODS}

Benzbromarone, brefeldin A (BFA), and bovine serum albumin (BSA) were purchased from Sigma (St. Louis, MO). PSC833 was a gift from Sandoz (Uden, The Netherlands). $\mathrm{C}_{6}$-NBD-ceramide (MBD-Cer) was from Molecular Probes (Eugene, OR). Cell culture filter supports were obtained from Costar (Cambridge, MA) and cell culture media were from Gibco (Paisley, UK). Chemicals and organic solvents were of analytical grade and obtained from Riedel-de Haën (Seelze, FRG).

\section{Cells}

LLC-PK1 pig kidney epithelial cells were cultured on filters glued to the bottom of a cup, so as to be able to separate apical and basolateral medium. LLC-PKI cells transfected with MDR1 P-glycoprotein, or with MRP1 were obtained from Alfred Schinkel and Raymond Evers, Netherlands Cancer Institute, Amsterdam (Schinkel et al., 1995; Evers et al., 1996; van Helvoort et al., 1996; Raggers et al., 1999). All cells used were free of mycoplasma contamination.

\section{Drug Incubations}

In order to block vesicular traffic, cells were preincubated with $1 \mu \mathrm{g} \mathrm{BFA} / \mathrm{ml}$ for $30 \mathrm{~min}$ at $37^{\circ} \mathrm{C}$, with $\mathrm{BFA}$ remaining present during subsequent incubations. Alternatively, transport incubations were performed at $15^{\circ} \mathrm{C}$ (van Helvoort et al., 1997). The activity of multidrug transporters was inhibited by preincubation for $10 \mathrm{~min}$ at $37^{\circ} \mathrm{C}$ with inhibitors (benzbromarone, $40 \mathrm{mM}$; PSC $833,5 \mu \mathrm{M}$ ), followed by a transport incubation in the continued presence of the inhibitors.

\section{Sphingolipid Transport Assay}

NBD-Cer was introduced into cells in Hanks' balanced salt solution buffered with $10 \mathrm{mM}$ Hepes, $\mathrm{pH} 7.2(5-15 \mu \mathrm{M})$ from BSA-complexes $(1 \% \mathrm{w} / \mathrm{w})$. Subsequent delivery of the newly synthesized NBD-GlcCer and NBD-SM to the outer leaflet of the plasma membrane was routinely assayed by continuous depletion by the BSA in 
the medium, either for $1 \mathrm{hr}$ at $37^{\circ} \mathrm{C}$ or for $3 \mathrm{hr}$ at $15^{\circ} \mathrm{C}$. This was followed by a $30 \mathrm{~min} 10^{\circ} \mathrm{C}$ wash with BSA (van Meer et al., 1987; van Helvoort et al., 1996; 1997). Apical and basal media of epithelial monolayers on filters were analyzed separately. Lipids were extracted from media and cells and, after two-dimensional separation on silica, NBD-lipid spots were visualized by UV light and quantitated by fluorometry (Jasco, Japan), all as described (van Helvoort et al., 1996).

\section{RESULTS AND DISCUSSION}

To answer the question whether transport of newly synthesized GlcCer from the cytosolic surface of the Golgi to the outer leaflet of the plasma membrane would be blocked in the absence of vesicular transport, we made use of a sphingolipid transport assay that is based on short-chain analogs of sphingolipids. It had been observed that a ceramide containing a $\mathrm{C}_{6}$-chain with a terminal fluorescent NBDmoiety when added to culture medium, enters cells and is converted to NBD-SM and NBD-GlcCer (Lipsky and Pagano, 1985). Upon reaching the cell surface, the analogs can be conveniently depleted from the outer leaflet of the membrane by BSA in the medium. At $37^{\circ} \mathrm{C}$ NBD-SM and NBD-GlcCer reach the cell surface, and in epithelial cells GlcCer was preferentially transported to the apical cell surface in contrast to SM yielding a difference in lipid composition similar to that observed in isolated apical and basolateral membranes (van Meer et al., 1987). This suggested that the analogs may be transported by the same mechanisms as natural SM and GlcCer.

When cells are treated with BFA, the Golgi fuses with the ER, and vesicular traffic to the plasma membrane is blocked. Indeed, newly synthesized SM no longer became available for hydrolysis by exogenous sphingomyelinase (Kallen et al., 1993; Shiao and Vance, 1993; van Helvoort et al., 1997). However, in contrast, newly synthesized NBD-SM still reached the cell surface (van Meer and van 't Hof, 1993). This puzzle was solved when we found that this BFA-insensitive transport of NBDSM to the outer leaflet of the plasma membrane was inhibited by a series of inhibitors of the multidrug transporter MDR1 P-glycoprotein (Pgp), like cyclosporin A, PSC 833, verapamil and progesterone (van Helvoort $e t$ al., 1997). In the presence of BFA, NBD-SM is synthesized in the lumen of the merged ER-Golgi. The available evidence suggests that lipids easily translocate across the ER membrane (Herrmann et al., 1990; Burger et al., 1996). Thus the NBD-SM would have access to the cytosol across which it could exchange with other membranes due to its enhanced "watersolubility". An MDR-like activity would then be responsible for its translocation across the plasma membrane. In support of this scenario, at $37^{\circ} \mathrm{C}$ in the absence of BFA, new NBD-SM is synthesized in the Golgi lumen and its transport to the cell surface is insensitive to the MDR-inhibitors and thus vesicular.

Transport of NBD-GlcCer to the cell surface was sensitive to MDR-inhibitors even in the absence of BFA, suggesting that its transport to the cell surface is nonvesicular (van Helvoort et al., 1997). Direct proof that MDR1 Pgp can translocate NBD-GlcCer and NBD-SM across plasma membranes was subsequently obtained in cells transfected with MDRI Pgp (van Helvoort et al., 1996). In these studies, vesicular traffic was abolished by performing the transport experiment at $15^{\circ} \mathrm{C}$. In 
contrast, transfer of NBD-lipids across the aqueous phase is not inhibited as exchange continues down to $0^{\circ} \mathrm{C}$ (van Meer et al., 1987). NBD-GlcCer reached the outside of the apical plasma membrane of epithelial LLC-PK1 cells when these cells had been transfected with MDR1 Pgp, but not in control cells. Transport was inhibited by MDR-inhibitors like PSC833. The closely related MDR3 Pgp, the putative transporter of the phospholipid phosphatidylcholine (PC) into the bile across the bile canalicular membrane of hepatocytes, was unable to transport NBD-ClcCer. Recently, we found that a relatively distant member of the ABC-transporter family, the multidrug resistance protein MRP1, can translocate NBD-GlcCer across membranes like MDR1 Pgp. However, MRP1 translocated NBD-GlcCer across the basolateral membrane of epithelial cells, and at $15^{\circ} \mathrm{C}$ this transport was inhibited by indomethacin and required glutathione (Raggers et al., 1999). The transport at $37^{\circ} \mathrm{C}$ was reduced to $50 \%$ by benzbromarone, an MRPI-inhibitor (Holló et al., 1996), but insensitive to the MDR 1-inhibitor PSC833 (Fig. 1). Apical NBD-GlcCer transport in these cells was most likely partially due to endogenous MDR1 Pgp as part of it was PSC833-sensitive. By changing the NBD-precursor to NBD-phosphatidic acid, which in the plasma membrane is converted to NBD-diacylglycerol (Pagano et al., 1981), we showed that NBD-PC is a substrate for MDR1 and MDR3 Pgp's, but not for MRP1. In contrast, NBD-phosphatidylethanolamine was a substrate for MDR1 Pgp only (van Helvoort et al., 1996).

What is the evidence that lipid translocation by ABC-transporters is of physiologic relevance? The connection of MDR3 Pgp to lipid transport was discovered when a knock-out mouse for the murine homolog of $M D R 3, M d r 2$, displayed a defect in transport of PC into the bile (Smit et al., 1993). Further evidence that MDR3 Pgp is the actual transporter for natural PC came from experiments demonstrating increased transport of natural PC to the cell surface of MDR3 Pgp-expressing mouse fibroblasts as monitored by its accessibility to exogenously added PCtransfer protein (Smith et al., 1994). Evidence for translocation of natural GlcCer by multidrug transporters is, so far, indirect. The amount of newly synthesized GlcCer in cells was reported to correlate with the expression level of MDRl Pgp (Lavie et al, 1996), and inhibitors of MDR1 Pgp function led to decreased levels of GlcCer (Lavie et al., 1997). One attractive idea would be that removal of GlcCer from the cytosolic surface of the plasma membrane, and probably other cellular membranes like endosomes and trans Golgi, relieves an inhibition on the level of GlcCer in that cytosolic leaflet. This may work by releasing feed-back inhibition of the ceramide glucosyltransferase, or by depriving substrate from a cytosolic cerebrosidase.

Our new evidence demonstrates the involvement of a non-lysosomal cerebrosidase in the low GlcCer content of cells without MDR activity (Raggers et al., unpublished data), suggesting that both a cytosolic cerebrosidase and a GlcCer translocator are involved in regulating the concentration of $\mathrm{GlcCer}$ in the cytosolic leaflet of cellular membranes. Interestingly, newly synthesized GlcCer appears to be essential for sorting the membrane enzyme tyrosinase from the Golgi to melanosomes in melanocytes and neutrons (Sprong et al., unpublished data). Its cytosolic orientation makes GlcCer a great candidate for creating lipid domains on the cytosolic surface of Golgi membranes. Maybe such cytosol-oriented domains are the 


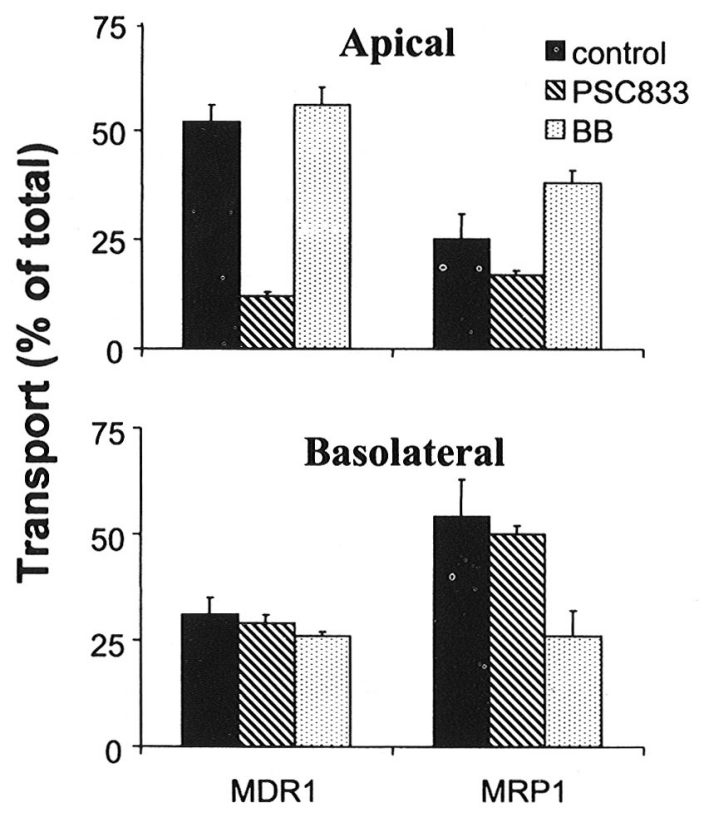

Fig. 1. Translocation of the $\mathrm{C}_{6}-\mathrm{NBD}$-analog of glucosylceramide across the two plasma membrane domains of LLC.PKI epithelial cells transfected with $M D R I$ or $M R P 1$. Cell monolayers on filters were incubated with BFA to inhibit vesicular traffic, with or without the MDR 1-inhibitor PSC833 and the MRP1-inhibitor benzbromarone (BB) as indicated. Subsequently, NBD-ceramide was added for $1 \mathrm{hr}$ at $37^{\circ} \mathrm{C}$, and transport of NBDglucosylceramide into the BSA-medium on either surface was measured, all as described under Materials and Methods. Transport is expressed as the percentage of total NBD-GlcCer that was found in either medium. NBD-GlcCer synthesis from $15 \mu \mathrm{M}$ NBD-Cer was $125 \mathrm{pmol}$ and $90 \mathrm{pmol}$ for the MDRI- and MRPItransfected cells, respectively, and was relatively insensitive to PSC $833(+15 \%)$ and benzbromarone $(-5 \%)$. Data represent the mean ( \pm s.d.; $n=4.6$ ) of 3 independent experiments.

counterpart of the luminal domains suggested many years ago as being involved in sorting of lipids and proteins to the apical surface of epithelial cells (van Meer $e t$ al., 1987; Simons and Ikonen, 1997). Removal of GlcCer from the cytosolic surface of a post-Golgi membrane may well be an intrinsic part of the Golgi sorting machinery. It would dissolve the sorting machinery and release the cargo in the next compartment. It is now important to know the intracellular localization of the nonlysosomal cerebrosidase (van Weely et al., 1993) and of the MDR1 Pgp in vivo. Further important questions are whether MDR1 Pgp is the translocator responsible for transport of GlcCer to the lumen of the Golgi, and whether the requirement of 
GlcCer for tyrosinase sorting reflects the involvement of the GlcCer in a general sorting pathway.

\section{ACKNOWLEDGEMENTS}

We would like to thank Piet Borst, Raymond Evers, Jan Wijnholds, Netherlands Cancer Institute, Amsterdam, Ardy van Helvoort, Numico, Wageningen, Peter van der Sluijs, University of Utrecht, Hans Aerts, University of Amsterdam, The Netherlands, and Yoshio Hirabayashi, Riken, Saitama, Japan, for reagents and cells and for their continued interest.

\section{REFERENCES}

Burger, K. N. J., van der Bijl, P., and van Meer, G. (1996) Topology of sphingolipid galactosyltransferases in ER and Golgi: Transbilayer movement of monohexosyl sphingolipids is required for higher glycosphingolipid biosynthesis. J. Cell. Biol. 133:15-28.

Coste, H., Martel, M. B., and Got, R. (1986) Topology of glucosylceramide synthesis in Golgi membranes from porcine submaxillary glands. Biochim. Biophys. Acta 858:6-12.

Evers, R. et al. (1996) Basolateral localization and export activity of the human multidrug resistanceassociated protein in polarized pig kidney cells. $J$. Clin. Invest. 97:1211-1218

Hakomori, S.-i. and Igarashi, Y. (1995) Functional role of glycosphingolipids in cell recognition and signaling. J. Biochem. 118:1091-1103.

Herrmann, A., Zachowski, A., and Devaux, P. F. (1990) Protein-mediated phospholipid translocation in the endoplasmic reticulum with a low lipid speciticity. Biochemistry 29:2023-2027.

Holló, Z., Homolya, L., Hegedủs, T., and Sarkadi, B. (1996) Transport properties of the multidrug resistance-associated protein (MRP) in human tumour cells. FEBS Lett. 383:99-104.

Kallen, K.-J., Quinn, P., and Allan, D. (1993) Effects of brefeldin A on sphingomyelin transport and lipid synthesis in BHK21 cells. Biochem. J. 289:307-312.

Keller, P. and Simons, K. (1998) Cholesterol is required for surface transport of influenza virus hemagglutinin. J. Cell Biol. 140:1357-1367.

Lannert, H., Bunning, C., Jeckel, D., and Wieland, F. T. (1994) Lactosylceramide is synthesized in the lumen of the Golgi apparatus. FEBS Lett. 342:91-96.

Lavie, Y., Cao, H. T., Bursten, S. L., Giuliano, A. E., and Cabot, M. C. (1996) Accumulation of glucosylceramides in multidrug-resistant cancer cells. J. Biol. Chem. 271:19530-19536.

Lavie, Y. et al. (1997) Agents that reverse multidrug resistance, tamoxifen, verapamil, and cyclosporin A, block glycosphingolipid metabolism by inhibiting ceramide glycosylation in human cancer cells. J. Biol. Chem. 272:1682-1687.

Lipsky, N. G. and Pagano, R. E. (1985) Intracellular translocation of fluorescent sphingolipids in cultured fibroblasts: Endogenously synthesized sphingomyelin and glucocerebroside analogues pass through the Golgi apparatus en route to the plasma membrane. J. Cell Biol. 100:27-34.

Lisanti, M. P. and Rodriguez-Boulan, E. (1990) Glycophospholipid membrane anchoring provides clues to the mechanism of protein sorting in polarized epithelial cells. Trends Biochem. Sci. 15:113-118.

Lisanti, M. P., Scherer, P. E., Tang, Z.-L., and Sargiacomo, M. (1994) Caveolae, caveolin and caveolinrich membrane domains: A signalling hypothesis. Trends Cell Biol. 4:231-235.

Okamoto, T., Schlegel, A., Scherer, P. E., and Lisanti, M. P. (1998) Caveolins, a family of scaffolding proteins for organizing "preassembled signaling complexes" at the plasma membrane. J. Biol. Chem. 273:5419-5422.

Pagano, R. E., Longmuir, K. J., Martin, O. C., and Struck, D. K. (1981) Metabolism and intracellular localization of a fluorescently labeled intermediate in lipid biosynthesis within cultured fibroblasts. $J$. Cell Biol. 91:872-877.

Raggers, R., van Helvoort, A., Evers, R., and van Meer, G. (1999). The human multidrug resistance protein MRP1 translocates sphingolipid analogs across the plasma membrane. J. Cell Sci. 112:415422. 
Schinkel, A. H., Wagenaar, E., van Deemter, L., Mol, C. A. A. M., and Borst, P. (1995) Absence of the mdrla P-glycoprotein in mice affects tissue distribution and pharmacokinetics of dexamethasone, digoxin, and cyclosporin A. J. Clin. Invest. 96:1698-1705.

Shiao, J.-Y. and Vance, J. E. (1993) Sphingomyelin transport to the cell surface occurs independently of protein secretion in rat hepatocytes $J$. Biol. Chem. 268:26085-26092.

Simons, K. and Ikonen, E. (1997) Functional rafts in cell membranes. Nature 387:569-572.

Smit, J. J. M. et al. (1993) Homozygous disruption of the murine $m d r 2$ P-glycoprotein gene leads to a complete absence of phospholipid from bile and to liver disease. Cell 75:451-462.

Smith, A. J. et al. (1994) The human MDR3 P-glycoprotein promotes translocation of phosphatidylcholine through the plasma membrane of fibroblasts from transgenic mice. FEBS Lett. 354:263-266.

van Helvoort, A., Giudici, M. L., Thielemans, M., and van Meer, G. (1997) Transport of sphingomyelin to the cell surface is inhibited by brefeldin $A$ and in mitosis, where $C_{6}-$ NBD-sphingomyelin is translocated across the plasma membrane by a multidrug transporter activity. J. Cell Sci. 110:75-83.

van Helvoort, A. et al. (1996). MDR1 P-glycoprotein is a lipid translocase of broad specificity, while MDR3 P-glycoprotein specifically translocates phosphatidylcholine. Cell 87:507-517.

van Meer, G. (1998) Lipids of the Golgi membrane. Trends Cell Biol. 8:29-33.

van Meer, G., Stelzer, E. H. K., Wijnaendts-van-Resandt, R. W., and Simons, K. (1987) Sorting of sphingolipids in epithelial (Madin-Darby canine kidney) cells. J. Cell Biol. 105:1623-1635.

van Meer, G. and van 't Hof. W. (1993) Epithelial sphingolipid sorting is insensitive to reorganization of the Golgi by nocodazole, but is abolished by monensin in MDCK cells and by brefeldin A in Caco-2 cells. J. Cell Sci. 104:833-842.

van Weely, S., Brandsma, M., Strijland, A., Tager, J. M., and Aerts, J. M. (1993) Demonstration of the existence of a second, non-lysosomal glucocerebrosidase that is not deficient in Gaucher disease. Biochim. Biophys. Acta 1181:55-62.

Varma, R. and Major, S. (1998) GPI-anchored proteins are organized in submicron domains at the cell surface. Nature 394:798-801. 